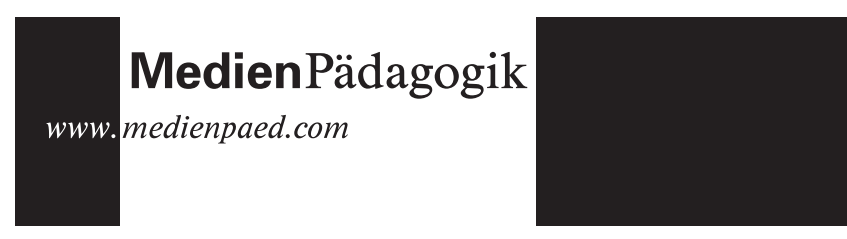

Burkhard Schäffer

23.3.2001

\section{Das Gruppendiskussionsverfahren in erziehungswissenschaftlicher Medienforschung}

Einleitung

Rar gesät sind Ansätze, die sich in Deutschland des Gruppendiskussionsverfahrens in Kontexten erziehungswissenschaftlicher Medienforschung bedienen. Dies ist von daher erstaunlich, weil sich Rezeption und Aneignung medial vermittelter Gehalte ${ }^{1}$, aber auch deren «Produktion» m. E. adäquat nur als Kollektivphänomene bzw. als kollektiv initiierte, inszenierte und enaktierte Prozesse konzeptualisieren lassen. So gewinnen z. B. in der Jugend- und frühen Erwachsenenphase Rezeption und Aneignung von Musik ihre Bedeutung erst in gruppenhaften Zusammenhängen, d. h. in der Institution der Peer-group, die sich vor dem Hintergrund ihrer je spezifischen kollektiven Erfahrungen mit dem medial Dargebotenen auseinander setzen (vgl. hierzu Schäffer 1996). Wie aus der Umfrageforschung hervorgeht, wird auch der Kinobesuch selten allein geplant, sondern gerade in der Jugendphase mit anderen Gleichaltrigen regelrecht inszeniert (vgl. Beer 2000, S. 66ff.). In derartigen kollektiven Kontexten bilden sich dann entsprechende Orientierungen hinsichtlich medialer Präferenzen aus und nicht - wie man angesichts der überwiegend individualisierenden Forschungspraxis annehmen könnte - im «stillen Kämmerlein〉 mit Fernsehanschluss. Gleiches gilt auch für die Praxis am Computer und <im〉 Internet, die m. E. ebenfalls nur verstanden werden können, stellt man die (bereits vorhandenen oder neu geschaffenen <virtuellen〉) sozialen Zusammenhänge in Rechnung (vgl. etwa Turkle 1995; Wetzstein 1995; Schäffer 2000).

${ }^{1}$ Zum Unterschied von Aneignung und Rezeption vgl. Mikos 2001
Pointiert lässt sich festhalten: Auch die Praxis des <einsamsten〉 Zeitungslesers, der <isoliertesten〉 Internetnutzerin oder des Fernseh-Vielsehers (Vitouch 1994) ist eingebunden in vielfache, kollektiv vermittelte «Spielräume und Rahmungen des Selbst» (Wittpoth 1994), die weit über die konkrete Medienpraxis des oder der Einzelnen hinaus weisen. Diese Spielräume und Rahmungen fungieren, etwa in milieu-, generations-, oder geschlechtsbezogenen Ausprägungen, als praktisches, oft implizites Orientierungswissen, das die Medienhandlungen leitet, ohne dass es den Medienhandelnden so bewusst ist.

In vielen Fällen mündet diese Erkenntnis gleichwohl nicht in eine entsprechende Forschungspraxis. Das methodische Repertoire, dessen man sich bedient, um kollektive Sachverhalte empirisch $\mathrm{zu}$ erfassen, ist überwiegend individualisierend geprägt. Anders und genereller formuliert: Obwohl sozialwissenschaftliche Sätze und Aussagen sich zumeist auf kollektive Sachverhalte beziehen bzw. auf Sachverhalte, die nur in ihrer Eingebundenheit in kollektive Strukturen zu erfassen sind, dominieren in der Forschungspraxis zumeist individualisierende Zugriffsweisen. Es wird überwiegend mit Einzelinterviews verschiedenster Ausprägungen gearbeitet: Von standardisierten, also 〈geschlossenen〉 über halbstandardisierte bis hin zu〈offenen〉 Verfahren, etwa narrativen oder biographischen Interviews.

Die genannten Beispiele mögen ausreichen, um den Argumentationsgang zu verdeutlichen: Viele Fragestellungen in Medienpädagogik, -forschung und -theorie befassen sich mit kollektiven Phänomenen und sind von daher dafür prädestiniert, mit einem Verfahren bearbeitet zu werden, das diese kollektiven Gehalte <einzufangen〉 vermag. Dies ist jedoch in der Praxis kaum der Fall. Es ist sicherlich nicht übertrieben zu behaupten, dass individualisierende Zugriffsweisen den Hauptanteil gegenwärtiger Sozialforschung ausmachen. Dies gilt auch über die engere medienpädagogische Forschung (vgl. Schorb 1998, S. 18f.) hinaus und zwar sowohl für die 〈qualitative Forschung〉 (vgl. exemplarisch Friebertshäuser/Prengel 1997; Flick/Kardorff/Steinke 2000) als auch für 〈quantitative〉 Ansätze (vgl. etwa Atteslander 1991, Kromrey 1998). Zwar macht es hinsichtlich der Entfaltungs- und Kommunikationsmöglichkeiten der Erforschten einen Unterschied, ob diese allein einen standardisierten Fragebogen ausfüllen sollen oder z. B. in einem biographisch-narrativen Interview zu ihrer Lebensgeschichte befragt werden. Gleichwohl handelt es sich in beiden Fällen um einen individualisierenden 〈Zugriff〉 auf den Forschungs- 
gegenstand: Die Beziehung zum kollektiven Charakter der untersuchten sozialen Phänomene wird in beiden Fällen erst nachträglich hergestellt. Bei einer Fragebogenuntersuchung geschieht dies, indem die Einzeläusserungen kategorisiert, aggregiert und dann zu kollektiven «Durchschnittsmeinungen hochgerechnet werden. In qualitativen Studien versucht man, durch eine grosse Tiefenschärfe das Exemplarische eines 〈Falles〉 herauszuarbeiten, dieses Exemplarische mit anderen Fällen zu vergleichen und so zu Aussagen zu gelangen, die über den Einzelfall hinaus auf 〈Kollektives〉 verweisen - sofern solch eine Analyse nicht ganz und gar der fallspezifischen Rekonstruktion verhaftet bleibt.

Eine andere Möglichkeit, die diesen individualisierenden Zugriff zu vermeiden hilft, besteht in der Verbindung von Einzeläusserungen mit dem vorausgesetzten kollektiven Charakter ihres Inhaltes. Sie verläuft über grundlagentheoretische Überlegungen. So wird beispielsweise im Anschluss an Mead auf die unhintergehbare Sozialität eines jeden Selbstbildungsprozesses verwiesen, allerdings besteht oft eine Diskrepanz zwischen grundlagentheoretischem Anspruch und deren forschungspraktischer Umsetzung.

Das Gruppendiskussionsverfahren leistet nun m. E. genau dies: Kollektive Orientierungen können sozusagen in 〈statu nascendi〉 herausgearbeitet werden. Gemeint ist hiermit nicht ein «direkter» Zugang zu kollektiv verteilten Orientierungen im Sinne von statistischen Häufigkeiten, sondern ein empirisch überprüfbarer Zugang zu kollektiven Phänomenen anhand einzelner Fälle (von Gruppen), ohne sich dabei in deren Singularität zu verlieren.

In diesem Beitrag werde ich zunächst einen kurzen historischsystematischen Überblick über einige wichtige Entwicklungslinien des Gruppendiskussionsverfahrens geben (1) und dann dessen Fruchtbarkeit für Fragestellungen im Kontext erziehungswissenschaftlicher Medienforschung am Beispiel eines konkreten Forschungsprojekts zu generationenspezifischen Mediennutzungs- und Aneignungskulturen herausarbeiten (2).

\section{Zur Entwicklung des Gruppendiskussionsverfahrens}

Die Entwicklung des Gruppendiskussionsverfahrens ${ }^{2}$ hat wiederholt wesentliche Impulse aus Kontexten der Medienforschung erhalten. In der Anfangszeit zu nennen sind hier vor allem die Studien von Merton und Kendall (1946) zur Erforschung von Zuschauerreaktionen auf Propagandafilme während des Zweiten Weltkrieges (Hoveland 1949 u. a., vgl. auch Lazarsfeld u. a. 1948). In dem sich hieraus entwickelnden «focus group interview» wurden Gruppen von Versuchspersonen «Grundreize» in Form von Produktverpackungen, Werbefilmen o. ä. vorgelegt und die Reaktionen der Gruppen, zumeist in Form von Wortbeiträgen, aufgezeichnet. Merton (1987), als Begründer des Focus-Group-Interviews, konzipierte das Verfahren jedoch fast ausschliesslich in seiner explorativen Funktion, gewissermassen als Appendix quantitativer Forschungsdesigns. Derartigen, vor allem im Bereich der Markt- und Konsumforschung nach wie vor dominierenden «survey sampling approaches», werden im angelsächsischen Sprachraum zunehmend auch Verfahrensweisen entgegengestellt, die mit «naturally occuring groups of like-minded people» (Livingstone/Lunt 1996, 82) arbeiten, also mit Realgruppen (vgl. etwa Liebes/Katz 1990).

Eine andere Bezeichnung (〈group discussions〉) und differentes Verständnis des Verfahrens finden sich vor allem im Kontext der 〈cultural studies〉, so im Bereich der Jugend- (vgl. exemplarisch Willis 1991), der Frauen- (vgl. Brown 1994, Gillespie 1995) und wiederum sehr intensiv in der Medienforschung. In letztgenannter war es vor allem Morley, der schon Anfang der 80er Jahre (vgl. Morley 1986) offene Diskussionen mit homogen zusammengesetzten Gruppen über das Fernsehprogramm «Nationwide» durchgeführt hatte, um herauszuarbeiten, wie Gruppen mit unterschiedlichem sozioökonomischem Hintergrund verschiedene «Lesearten» ein und desselben Fernsehprogrammes entfalteten.

\footnotetext{
2 Eine Gruppendiskussion ist nicht mit einer Gruppenbefragung und auch nicht einem Gruppengespräch zu verwechseln. Bei Gruppenbefragungen oder auch Gruppeninterviews (Atteslander 1991, 174) handelt es sich lediglich um zeitökonomische Varianten der Einzelbefragung. Die Gruppe als solche wird weder methodisch noch methodologisch als Gegenstand der Erhebung konzipiert. Hiervon abzugrenzen sind «natürliche〉 Gesprächssituationen, an denen vor allem die Konversationsanalyse ein Interesse hat (vgl. exemplarisch: Luckmann 1986; Günthner/Knoblauch 1994;
} Bergmann 1994; Keppler 1994; Hepp 1998). 
Das Gruppendiskussionsverfahren hatte und hat in der empirischen Sozialforschung in den USA und in Grossbritannien einen hohen Stellenwert inne. Dies zeigt sich nicht zuletzt daran, dass es eine stattliche Anzahl an Einführungs- und 〈how to do it>-Literatur in englischer Sprache gibt, die sich hinsichtlich methodologischer Fragen allerdings zumeist recht abstinent zeigt (vgl. exemplarisch Greenbaum 1993; Morgan/Krueger 1998).

In Deutschland wurde das Gruppendiskussionsverfahren einer breiteren Öffentlichkeit Mitte der 50er Jahre vorgestellt. Im Zuge einer am Frankfurter Institut für Sozialforschung durchgeführten Studie zum politischen Bewusstsein im Nachkriegsdeutschland wurde eine «Gruppentechnik» zur «Ermittlung von Meinungen in statu nascendi» (Pollock 1955 , S. 32) entwickelt und von Pollock rekonstruiert. In der für die Frankfurter Schule typischen, psychoanalytisch geprägten Empirie ging es darum, hinter «Abwehrmechanismen und Rationalisierungen» zu schauen. Unbeschadet der theoretischen Kritik an der Isolierung indi-vidueller Meinungen in der Umfrageforschung wurden in der (quantitativen) empirischen Auswertung die einzelnen Redebeiträge dann aber doch isoliert voneinander analysiert. In dieser Tradition entstanden z.T. sehr einflussreiche Studien, etwa im Bereich der Bildungsforschung (Schulenberg 1957) und wiederum auch in der Medienforschung (Maletzke 1959).

In bezug auf eine Weiterentwicklung des Gruppendiskussionsverfahrens ist jedoch vor allem die ebenfalls am Frankfurter Institut entstandene Arbeit von Werner Mangold zu nennen (Mangold 1959; vgl. auch: Ders. 1973). Sie stellt unter methodologischen und forschungspraktischen Gesichtspunkten den Abschluss und Höhepunkt der Entwicklung des Gruppendiskussionsverfahren bis weit in die 70er Jahre hinein dar. Mit seinem Konzept der «informellen Gruppenmeinungen», die sich in «sozialen Grossgruppen» ausbildeten, eröffnete Mangold die Perspektive eines Paradigmenwechsels: Wurden Gruppendiskussionen bis zu diesem Zeitpunkt schwerpunktmässig eingesetzt unter dem Aspekt der besseren Ermittlung der Meinungen und Einstellungen Einzelner unter Gruppenkontrolle, so bereitete Mangold das Terrain für die Erforschung kollektiv verankerter Orientierungen.

Erst gegen Ende der 70er Jahre wurde die methodisch-methodologische Diskussion über das Gruppendiskussionsverfahren fortgeführt. Beeinflusst von der AG Bielefelder Soziologen (1973) und unter Rückgriff auf Konzepte des Symbolischen Interaktionismus sowie der phänomeno- logischen Soziologie leisteten Nießen (1977) und Volmerg (1977) einen wesentlichen Beitrag zur Weiterentwicklung des Verfahrens. Mitte der 80er Jahre wurde das Gruppendiskussionsverfahren dann vermehrt von der Jugendforschung aufgegriffen. Behnken (1984) und Peukert (1984) beschäftigten sich am «Institut für Jugendforschung und Jugendkultur» mit der Weiterentwicklung des Verfahrens als «Methode zur Rekonstruktion der Lebenswelt von Lehrlingen». Zur selben Zeit, Mitte der 80er Jahre, entwickelte Bohnsack, zunächst in Zusammenarbeit mit Mangold, das Gruppendiskussionsverfahren weiter zu der hier vertretenen Methode (vgl. Mangold/Bohnsack 1988, Bohnsack 1989 und 2000a, siehe auch Loos/ Schäffer 2001).

Entscheidend für diesen neuen Ansatz ist vor allem der Einbezug der sog. «Dokumentarischen Methode» bei der Auswertung der Gruppendiskussionsprotokolle (Mannheim 1964; vgl. auch Bohnsack 1989, ders. 1997, ders. 2000a). In dieser Perspektive wird die konkrete Gruppe als Epiphänomen bzw. als Dokument für milieu-, geschlechts- und generationsspezifische «konjunktive Erfahrungsräume» konzipiert (Mannheim 1980, S. 211ff.). Ein konjunktiver, also verbindender, Erfahrungsraum basiert nach Mannheim auf gemeinsamen biographischen und kollektivbiographischen Erlebniszusammenhängen (a.a.O., S. 271) derjenigen, die diesem Erfahrungsraum angehören. Hierzu gehört neben geschlechts-, milieu- und bildungsspezifischen Erfahrungsräumen auch die Erfahrung der Zugehörigkeit zu einer Generation. Bei der Interpretation einer Gruppendiskussion im Rahmen der dokumentarischen Methode stehen also weder die Meinungen und Einstellungen der Einzelnen, noch die situative Prozesshaftigkeit des Austausches von Argumenten im Mittelpunkt der Aufmerksamkeit der Interpretierenden. Vielmehr werden jetzt die sich auf einer oberflächlichen Ebene von Situation zu Situation unterscheidenden und insofern scheinbar emergenten, interaktiven Phänomene einer Gruppendiskussion in ihrer Repräsentanz für kollektive Prozessstrukturen begriffen (Loos/Schäffer 2001, S. 101). Der am häufigsten geäusserte Kritikpunkt am Gruppendiskussionsverfahren, seine fehlende Reliabilität, also die Wiederholbarkeit und damit Vergleichbarkeit ähnlicher Thematiken (vgl. etwa bei Kromrey 1986, S. 128ff.; Spöhring 1989, S. 216; Lamnek 1998, S.74ff.), wird hierdurch entkräftet, denn: Eine Gruppe kann sich, um das zur Artikulation zu bringen, was ihren kollektiven Bezug ausmacht der unterschiedlichsten Themen bedienen: Auf einer immanenten Ebene ist es für einen Interpretierenden nicht möglich, einen Sinn aus der 
unablässigen Abfolge der Themen und Beiträge in einer Gruppendiskussion zu erschliessen. Auf einer dokumentarischen Ebene dagegen artikuliert die Gruppe gewissermassen von Thema zu Thema präziser <ihr> «kollektives Orientierungsmuster» (Bohnsack 1997), das auf die kollektiv geteilten Erlebniszusammenhänge der Gruppenmitglieder verweist, welche ihrerseits die konjunktiven Erfahrungsräume fundieren. Entsprechend kann dann auch nicht mehr von einer Gruppe gesprochen werden, wenn es sich in einer Diskussion zeigt, dass deren Mitglieder über keinerlei Gemeinsamkeiten bei den Erfahrungshintergründen verfügen.

Im engeren und weiteren Kontext dieser Konzeptualisierung des Gruppendiskussionsverfahrens sind in der jüngsten Zeit eine Fülle empirisch angelegter Studien entstanden, die die Leistungs- und Anschlussfähigkeit dieses Ansatzes in unterschiedlichen Anwendungsgebieten unter Beweis stellen. ${ }^{3}$

Im anschliessenden Kapitel wird der Einsatz des Gruppen-diskussionsverfahrens exemplarisch anhand eines laufenden Forschungsprojekts zur «Aneignung von Computerwissen innerhalb generationenspezifischer Mediennutzungs- und Aneignungskulturen» skizziert.

\section{Forschungsbeispiel für eine Gruppendiskussion in der erziehungs- wissenschaftlichen Medienforschung: Die Aneignung von Computer- wissen innerhalb generationenspezifischer Mediennutzungs- und Aneignungskulturen}

Für medienpädagogische und -andragogische Kontexte gleichermassen interessant ist die Frage nach der Aneignung von Computerwissen in unterschiedlichen Altersgruppen. «Computerbildung» bzw. «computer literacy» stellen sowohl im Kindes- und Jugendalter als auch für Prozesse lebenslangen Lernens bzw. lebenslange Bildungsprozesse zunehmend eine wesentliche Voraussetzung gesellschaftlicher Teilhabe dar (vgl. Moser 1999, Schorb 1995). Gleichzeitig lassen sich am Beispiel der Aneignung von Computerwissen Generationenphänomene studieren, da kaum eine andere Wissensform einem derartig schnellen Wandel unterworfen ist und für weite Kreise in der Bevölkerung hohe und bewusste Alltagsrelevanz besitzt.

\footnotetext{
3 Vgl. exemplarisch: Bohnsack 1989, Bohnsack u. a. 1995, Bohnsack/Nohl 1998 Schäffer 1996 sowie 1998a, 1998b u. 2000a, Nohl 1996 und 2001, Loos 1998 und 1999, Meuser 1998, Nentwig-Gesemann 1999, Breitenbach 2000, Michel 2001.

In der Studie wurden Gruppendiskussionen mit computerinteressierten Gruppen unterschiedlichen Durchschnittsalters durchgeführt, um die impliziten Bedeutungszusammenhänge herauszuarbeiten, an denen sich die medienbezogene Handlungspraxen der unterschiedlichen Altersgruppen und damit auch deren Formen und Stile der Aneignung von Computerwissen orientieren. Diese Formen handlungsleitenden Orientierungswissens werden in der Untersuchung als «Mediennutzungskulturen» konzeptualisiert und es wird zwischen deren milieu-, geschlechts-, bildungs- und vor allem ihrer generationensspezifischen Ausprägung unterschieden (vgl. hierzu Schäffer 1998a und b sowie 2001).

\subsection{Sample}

Seit 1998 wurden in einer Mittelstadt in Ostdeutschland Gruppendiskussionen mit insgesamt 15 computerinteressierten Gruppen durchgeführt, von denen als empirische Eckfälle zentral 8 Gruppendiskussionen bearbeitet werden. Grob lassen sich die Gruppen in 〈Junge〉 (16 Jahre, zumeist Schüler), 〈Mittelalte〉 (Berufstätige um die 40 Jahre) und 〈Alte〉 (Besucher von Computerkursen für Senioren um die 65 Jahre) unterteilen. Die Gruppen sind «Realgruppen», d. h. sie wurden nicht eigens für die Diskussion zusammengerufen, sondern kennen sich aus anderen Kontexten. Sie sind z. T. geschlechtshomogen, z. T. gemischt-geschlechtlich zusammengesetzt. Gerade bei den älteren Teilnehmenden unterscheiden sich die Mitglieder der Gruppen nach Herkunft, Bildungsstand und Beruf in erheblicher Weise. Dieser beabsichtigten Heterogenität des Samples stand ein homogener Faktor gegenüber, der für die Fragestellung - neben dem Alter - als tertium comparationis dient: Alle Gruppen haben Umgang mit Computern und für fast alle Gruppen gilt, dass der Computer auch ein wichtiger bzw. der einzige Anlass ist, sich zu treffen. Mit einer bewusst unscharf gehaltenen Eingangsfrage nach Altersunterschieden bei der Mediennutzung gelang es, in allen Gruppen selbstläufige Diskurse anzustossen, die sich einerseits im Spannungsfeld zwischen der Materialität der Technik, ihrer medialen Thematisierung («Computerkids», «Senioren im Netz») und dem erfahrungsgebundenem Wissen über die Technologie bewegten. Zum anderen aktualisierten die Gruppen anhand des Themenbereichs «Neue Medien» explizit und implizit verschiedene Dimensionierungen der Generationenthematik. 
2.2 Fallbeispiel: Die Gymnasiastengruppe Kermit

Im folgenden soll nun anhand der Eingangssequenz einer Gruppendiskussion mit einer jungen Gruppe des Samples einige Aspekte des bisher nur theoretisch Explizierten beispielhaft veranschaulicht werden. Die Gruppe trägt den Codenamen Kermit und besteht aus 5 Gymnasiasten im Alter von 16 bis 17 Jahren. Die Eingangsfrage ist aus Demonstrationszwecken mit aufgenommen: ${ }^{4}$
Y: Medien (.) immer wieder berichtet dass es Altersunterschiede in bezug auf die Nutzung von Medien gibt. Also alt und jung (.) ähm was weiss ich sie kennen ja selber bestimmt Berichte über Computerkids oder Senioren im Netz oder wie auch immer die denn immer übertitelt werden und wenn sie sich nun mal einfach mal daran erinnern oder wenn sie einfach auch darüber erzählen wie ihre ganz persönliche Erfahrung im Augenblick is ähm fallen ihnen hierzu Begebenheiten oder Geschichten ein wo es rings um die Nutzung von Medien geht in diesem Zusammenhang von alt und jung also Medien ganz allgemein Videorecorder Computer

Dm: Lhmm

Lach so

Y:

also erst mal so ganz breit gefasst

$\mathrm{Bm}:$ alt und jung also alles von uns bis siebzig hoch denn?

Lna ja was sie selber und da eben auch vor allen Dingen was sie auch selber für Erfahrungen damit gemacht haben

Dm: @also@

Na ja gerade mit Computern

?m:

$$
\text { L@(.)@ }
$$

4 Zur Transkription: Die Gruppenmitglieder werden mit den Anfangsbuchstaben des Alphabets bezeichnet; den männlichen Teilnehmenden wird das Kürzel〈 $\mathrm{m} 〉$, den weiblichen ein $\langle\mathrm{f}\rangle$ hinzugefügt. Interviewende tragen immer den Buchstaben $\langle\mathrm{Y}\rangle$. Durch das Zeichen $\langle @\rangle$ eingerahmte Satzteile oder Worte zeigen an, dass diese Worte oder Satzteile lachend gesprochen werden. @(.)@ bedeutet kurzes Auflachen. Das Zeichen $L$ zeigt den Beginn einer Überlappung an (vgl. zum verwendeten Transkriptionssystem Loos/Schäffer 2001, S. 55).
Dm:

Lmeine Eltern die sind nicht ganz so auf der Höhe äh was Computer@betrifft@ und es is ähm na ja also viel Ahnung da is da nich also meine Mutter arbeitet mit dem Computer und hat da richtig nen Arbeitscomputer aber

Bm: L( )

L da wird halt nicht viel gemacht irgendwie. Also da is nen Winword und ähm Na ja denn denn wird halt dis Winword benutzt und dis sieht man denn als dis Betriebssystem
Bm:

Dm:

$$
\text { Ł@ja wirklichja@ }
$$

$L$ und denkt also auch mehr is da nich` zu machen man hat da schreibt seine Texte und drückt denn auf senden und ähm (.) find ich eigentlich irgendwie traurig weil mit'm Rechner kann man viel mehr machen und das weiss ich jetzt ja selber auch und äh ja so sieht das denn bei mir aus zu Hause

Durch die Eingangsfrage (und natülich den Vorlauf vor der Diskussion) werden spezifische thematische Vorgaben gemacht bzw. Diskussionsanlässe geschaffen, auf die die Gruppen in ihrer je spezifischen, ihrem Habitus bzw. ihrem modus operandi entsprechenden Form reagieren. Die vorliegende Eingangsfrage birgt z. B. das Potenzial in sich, eine Auseinandersetzung zwischen erfahrungsgebundenem (milieu-spezifischem) Wissen und medial vermitteltem Wissen in die Gruppe 〈hineinzutragen〉. In der Frage wird eine implizite Hypothese formuliert, die auf die Diskrepanz zwischen selbsterlebten Erfahrungen mit Neuen Medien einerseits und medial konstruierten Sachverhalten andererseits abhebt - verkörpert in den medial vermittelten Konstrukten von 〈Computerkids〉 oder 〈Senioren im Netz>.

Generell gehört es zu unseren methodischen Prinzipien, die Eingangsfrage möglichst <ungenau〉 bzw. offen zu formulieren (vgl. Bohnsack 1999, S. 212ff.; Loos/ Schäffer 2001, S. 49ff.), so dass die Gruppen sich diejenigen Aspekte der Frage gewissermassen heraussuchen können, die für sie noch am ehesten mit ihrer Erfahrung in Verbindung stehen. Dies tun die Mitglieder der Gruppe Kermit schon während der Frageformulierung (die Präzisierungsnachfrage von $\mathrm{Bm}$ ). In der ersten Reaktion nach Abschluss der Formulierung der Eingangsfrage von $D m(« \mathrm{Na}$ ja gerade mit Computern@also@») und dem Lachen von?m passt die Gruppe die Frage 
dann ihrem Relevanzsystem an. Darüber hinaus dokumentiert sich, dass die in dieser Form gefasste Thematik als solche angenommen, also ratifiziert wird: Es ist ein Thema, über das man etwas zu sagen hat und es ist auch eine Thematik, bei der $D m$ offensichtlich sicher sein kann, sich mit den anderen über strukturidentische Erfahrungen austauschen zu können: Das indexikale, also für einen Beobachter nicht eindeutige und nur den Gruppenmitgliedern verständliche Lachen beim «also» wird zumindest von $? m$ erwidert. Es drängt sich der Eindruck auf, dass $D m$ eigentlich nicht weiter zu exemplifizieren bräuchte, damit die anderen ihn verstehen.

Nach dieser Präambel wird das Thema dann endgültig in die Richtung gebracht, die den Relevanzen der Gruppe entspricht: Am Beispiel Computer zeigt sich der Unterschied alt und jung am deutlichsten und zwar im familiären Nahraum, wie Dm ausführt. Es sind also auch noch andere Neue Medien und andere Kontexte vorhanden, bei denen sich diese Problematik stellt, beim Computer in der Familie tritt sie ganz besonders zu Tage. Das Problematische bei den Eltern liegt in den Augen der Gruppe denn für diese spricht $D m$ hier $^{5}$ - in deren Wissen über den Computer («viel Ahnung ist da nicht»). Die Eltern sind aus der Perspektive der Gruppe nicht in der Lage, zwischen Oberfläche und Systemtiefe zu unterscheiden: Sie halten ein Schreibprogramm («Winword») für das Betriebssystem. Das dem Computer innewohnende Potenzial wird von den Eltern auf Grund ihres Unwissens nicht erkannt, und das ist «traurig».

$D m$ bezieht sich hier stellvertretend für die anderen auf seine Eltern gewissermassen im Modus eines Erwachsenen, der, aus Erfahrung klug geworden, 〈verzweifelt〉 auf sein Kind schaut, das die guten Ratschläge nicht befolgen will, von denen er weiss, dass sie richtig für das Kind sind Hierauf deutet auch die Formulierung «und das weiss ich jetzt ja selber» hin. Sie verweist darauf, dass ihm bewusstseinsmässig sehr wohl ein Zustand vor und einer nach der Initiation durch die Computerpraxis und die Gruppe präsent ist. Wir haben es also mit einer Umkehrung der üblicherweise verhandelten Beziehungen im Lebenszyklus zu tun. D m bezieht sich auf seine Eltern offensichtlich partiell wie ein Erwachsener auf sein Kind bzw. wie ein Erzieher auf zu Erziehende. Vor dem Hintergrund der offensichtlich lebenszyklisch geprägten kollektiven Orientierung der Gruppe - denn eine solche Orientierung dokumentiert sich hier - ist es

\footnotetext{
5 An den Validierungen der anderen dokumentiert sich, dass er hier eine kollektiv geteilte Erfahrung artikuliert.
}

$11 / 20$

«traurig», dass die Eltern keinen Wissensvorsprung innehaben. Überspitzt formuliert: Die Eltern müssten sich eigentlich besser als sie selbst auskennen in den Programmen, da dies in einem lebenszyklischen Rahmen ihrer naturwüchsigen Position entspräche. Nähme die Gruppe dagegen eine Generationenposition ein, wäre es nur normal, dass die Eltern sich nicht auskennen, da es in einer Generationenperspektive zu erwarten ist, dass die Jüngeren sich neue Wissensbestände aneignen, die den Alten (oft aus habituellen Gründen) nicht mehr zugänglich sind. Die Jugendlichen beklagen also gewissermassen ihren eigenen Wissensvorsprung; implizit sind sie an einer lebenszyklischen «Normalverteilung〉 von Wissen orientiert.

In der sich daran anschliessenden Thematisierung der Grosselterngeneration wiederholt sich dieses Schema, nun jedoch bezogen auf die Bedienung von Video- und Fernsehgeräten:

Bm: Das fängt ja schon bei dis is ja nich nur bei Computern so zum Beispiel meine Oma die die hat nich mal Ahnung vom Fernseher die weess' wie man umschaltet laut und leise stellt und das war's

?m:

Dm:

Bm:

Dm:

Bm:

Auch die Grosseltern können ihnen hier nichts zeigen, im Gegenteil: Für diese müssen die aus der Sicht der Gruppe einfachsten Bedienungshandgriffe erledigt werden. Wollte man ihnen etwas beibringen, müsste die Bedienungsanleitung benutzt und auch auf deutsch übersetzt werden. Am schliesst hieran unmittelbar mit folgendem Beitrag an:

L Na bei mir zu Hause is es auch so ähnlich also meine Eltern benutzen Computer jetze beruflich zwar auch aber ähm weniger Schreibarbeit sondern mehr so Maschinensteuerung oder irgend 
so was in der Art oder na ja meine Mutter nützt ooch noch zum zum zum Schreiben jetze Arztpraxis aber zu Hause jetzt nich irgendwo berufliche Nutzung noch nebenbei sondern ähm hauptsächlich (.) auch hin und wieder mal nen Spiel und auch ins Internet wolln se hin und wieder aber dann meistens auch nur um irgendwas zu erledigen was machen müssen also um Buch einkaufen oder Auktion oder irgendsowas in der Art aber jetzt weniger so mal das blosse Stöbern (.) uuund (.) na ja meine Grosseltern ham` also meine Oma hat nen Computer die benutzt den auch hauptsächlich um na ja Briefe schreiben bisschen Computerspiele ähm also denn (.) Geschicklichkeitsspiele oder sonstwas also setzt sich jetzt nich irgendwo hin und

?m:

Em:

Am:

Em:

Am:

$\mathrm{Bm}:$

Am:

Em:

$\mathrm{Am}$

$\mathrm{Dm}$

Am:

$\mathrm{Cm}$ :

me:

Am:

$\mathrm{Cm}:$ was was spieln die denn so?

$$
\text { was? }
$$

was spieln die denn Herz oder irgendwie so was

Lna ja da Herz und

denn gibt's da irgendwie noch son Auf- so etwas über Shareware L(schlägt )

auch noch Adventurespiele (.) also (.)

$$
\text { LCommanderKey@(.)@ }
$$

$$
\text { also (.) }
$$

Lzu brutal

L irgendwas schönes mit Rätseln auch mal mit der 3DUmgebung aber (.) im Endeffekt (.)

Lauch mal ne Cyberbrille @(.)@

$$
\text { L@(.)@ }
$$

Lmeine andere Oma die ham sich

zwar mal überlegt jetzte n'Rechner anzuschaffen aber da ging es im Endeffekt nur darum Telefonkosten nach Amerika zu sparen um Briefverkehr zu beschleunigen da ging's im Endeffekt nur ums Internet aber ham se jetzt auch so' $n$ bisschen verworfen weil se einfach der Meinung sind a'dass se sich da nich schnell genug reinfinden würden uund (.)

Lsterben eher als dass se es kapiern“ auszudrücken

$A m$ berichtet von einer ziemlich ausgeprägten Computerpraxis seiner Eltern und seiner Grosseltern, zieht jedoch eine Parallele zu seinem Vorgängerbeitrag: «Na bei mir zu Hause is es auch so ähnlich». Diese scheinbare Diskrepanz - denn die Eltern arbeiten ja mit dem Computer im Beruf und zu Hause und benutzen ihn im Internet, sogar die Grosseltern besitzen einen Computer - diese Diskrepanz findet ihre Erklärung darin, dass die Jugendlichen an die Eltern die Erwartung hegen, dass diese den Computer in einer ihnen ähnlichen Art und Weise benutzen. Dass sie also z. B. im Internet nicht nur zweckrational motiviert nach etwas Bestimmten suchen oder etwas kaufen, sondern sich dem «blossen Stöbern» hingeben bzw., dass sie nicht nur die einfachen Spiele spielen, sondern auch in den Augen der Gruppe anspruchsvollere Anwendungen ausprobieren. Dabei wissen sie, dass die Eltern bzw. Grosseltern natürlich nie «Commander key» ${ }^{6}$ spielen bzw. eine «Cyberbrille» aufsetzen würden. Die Vorstellung erscheint ihnen lächerlich, was darauf verweist, dass sie auch bei Spielen recht genaue Kriterien dafür haben, was altersangemessen ist und was nicht. Sie befinden sich hier in einer klassischen Dilemmasituation: einerseits ist es «traurig», dass die Eltern nicht wissen, was man alles mit dem Computer machen kann (vgl. w.o.), andererseits würden sie es aber als altersunangemessen ansehen, wenn die Eltern wirklich all das machen würden, was sie selbst mit dem Computer machen. Sie stossen sich hier gewissermassen an den Aporien ihres impliziten lebenszyklischen Modells, nach dem es einerseits ein wissensbezogenen Normalabstand zwischen den Altersgruppen einzuhalten gilt (Motto: Die Eltern wissen mehr und wir Jüngere versuchen sie einzuholen) und es andererseits lebenszyklisch bedingte Phasen gibt, in denen man, ohne Verdacht zu erregen oder als lächerlich angesehen zu werden, «Commander key» spielen bzw. eine «Cyberbrille» aufsetzen kann. Das heisst aber auch, dass in dieser Gruppe ein kollektives Wissen darüber vorhanden ist, wie man sich Computerwissen auf eine Art und Weise aneignet, die zu einer inkorporierten, selbstverständlichen und damit habitualisierten Form des Wissens führt (vgl. Tully 2000). Und genau dies beurteilen sie als «traurig», da sie

\footnotetext{
${ }^{6}$ Commander key ist ein Weltraumschlachtenspiel, das sich an Star Wars anlehnt.
} 
wissen, dass ihre Eltern nie zu diesem selbstverständlichen, mühelosen Umgang mit dem Computer gelangen werden, wie es ihnen selbst möglich ist. Von dieser kollektiven Orientierungsfigur ist, versichert man sich der anderen hier nicht wiedergegebenen weiteren Passagen, ein grosser Teil ihrer Bezugnahme auf die Elterngeneration geprägt.

Der Zynismus bzw. der aggressive Unterton, der sich in der These «die sterben eher als bis se das kapieren» ausdrückt, kann auf der Grundlage der Interpretation dieses einzelnen Falls zunächst einmal nur als ein Dokument für die enttäuschte Erwartung der Jugendlichen angesehen werden, dass die erwachsenen Verwandten die ambivalenten lebenszyklischen Vorstellungen der Jugendlichen nicht erfüllen (bzw. nicht erfüllen können, denn wie sie es auch machen, machen sie es ja falsch). Normalerweise brich sich dieser Zynismus oder auch die Arroganz, wie aus dem Vergleich mit anderen Fällen hervorgeht, nur dort Bahn bzw. ist nur dort nicht «traurig» gefärbt, wo es nicht um Verwandte geht, z. B. in bezug auf Lehrer und Lehrerinnen und deren mangelnde Computerkompetenz.

So weit zu einer exemplarischen Analyse einer Eingangssituation einer Gruppendiskussion. In einem nächsten Schritt des Forschungsprozesses sind dann Vergleichshorizonte mit anderen Gruppen heranzuziehen Hierbei gilt es, über die Technik maximaler und minimaler Kontraste einzelne Fälle gegeneinander zu halten und sukzessive zu generalisierenderen Aussagen zu gelangen (Typenbildung): Wie bearbeiten also gleichaltrige, aber vom Bildungsmilieu oder vom Geschlecht her sich unterscheidende Gruppen diskursiv die Thematik «Neue Medien und Familie», an der sich, wie ansatzweise herausgearbeitet werden konnte, kollektive Orientierungsmuster zum Bereich «Lebenszyklus oder Generation?» dokumentieren? Und weiter: Wie bearbeiten die mittelalten und die alten Gruppen diese semantischen Felder? Welche Funktionalität (Mannheim) haben für sie die Neuen Medien? In welchen Kontexten gewinnen die Medien für sie Sinnhaftigkeit und in welchen nicht und wie unterscheiden sie sich hier wiederum von den jungen Gruppen? Auf diese Weise bildet sich ein immer engeres Verweisungsgeflecht heraus, das mit Matthes als «Denkraum» (Matthes 1992) bezeichnet werden und auf dessen Grundlage eine Typologie generationsspezifischer Mediennutzungskulturen erstellt werden kann.

\section{Zusammenfassung: Gruppendiskussionen als Epiphänomene kollektiver Orientierungmuster}

An dem Beispiel sollte die zuvor beim historisch-systematischen Durchgang herausgearbeitete These verdeutlicht werden, dass die Gruppe in der hier präferierten Konzeptualisierung des Verfahrens für Interpretierende nicht als Gruppe interessant ist, sondern als Epiphänomen für die Artikulation kollektiver Orientierungsmuster, in denen sich unterschiedliche konjunktive Erfahrungsräume dokumentieren. Mit dem Instrumentarium der Gruppendiskussion sind diese Erfahrungsräume rekonstruierbar und es lassen sich vor allem - und hier wird es für Medienpädagogik und -andragogik interessant - die Funktionalität der verschiedenen Medien innerhalb dieser Erfahrungsräume präzise aufzeigen. In den gemeinsamen Erzählungen und Beschreibungen der Gruppen dessen, was sie, in welchen Zusammenhängen und vor allen Dingen: wie (!) mit den Neuen Medien machen, schält sich behutsam und manchmal unspektakulär das heraus, was ich als Mediennutzungskulturen bezeichnet habe. Um diese Kulturen in ihrer Vielfältigkeit und vor allem in ihrer Eingebundenheit in milieu-, geschlechts- und generationsspezifische kollektive Orientierungsmuster zu rekonstruieren, ist das Gruppendiskussionsverfahren als ein hochgradig valides Instrument anzusehen. Mit ihm kann darüber hinaus die eingangs beschriebene forschungspraktische Fixierung auf das Individuum <aufgebrochen > bzw. relationiert werden. Von hier aus können m. E. neue Impulse für eine grundlagenorientierte erziehungswissenschaftliche Medienforschung ausgehen, die die forschungspraktische Dichotomie zwischen «Individuum» und «Kollektiv» zwar nicht aufheben, aber zumindest 〈lindern $>$ helfen. 


\section{Literatur}

Arbeitsgruppe Bielefelder Soziologen (Hrsg.)(1973): Alltagswissen, Interaktion und gesellschaftliche Wirklichkeit. Reinbek bei Hamburg, 2 Bde. (Neuauflage 1980, Opladen).

Atteslander, P. (1991): Methoden der empirischen Sozialforschung, Berlin, New York ${ }^{6}$.

Beer, C. (2000): Die Kinogeher. Eine Untersuchung des Kinopublikums in Deutschland. Berlin.

Behnken, I. (1984): Jugendbiographie und Handlungsforschung. Gruppendiskussionen als Methode zur Rekonstruktion der Lebenswelt von Lehrlingen. Band II, Frankfurt/M.

Bergmann, J. R. (1994): «Ethnomethodologische Konversationsanalyse». In: Fritz, G. und Hundsnurscher, F.: Handbuch der Dialoganalyse. Tübingen.

Bohnsack, R. (1989): Generation, Milieu und Geschlecht. Opladen.

Ders. (1997): «Orientierungsmuster: Ein Grundbegriff qualitativer Sozialforschung». In: Schmidt, F. (Hg.): Methodische Probleme der empirischen Erziehungswissenschaft. Baltmannsweiler.

Ders. (1998): «Milieu als konjunktiver Erfahrungsraum. Eine dynamische Konzeption von Milieu in empirischer Analyse». In: Matthiesen, U. (Hrsg.): Die Räume der Milieus. Neue Tendenzen in der sozial- und raumwissenschaftlichen Milieuforschung in der Stadt- und Raumplanung. Berlin.

Ders. (2000a): Rekonstruktive Sozialforschung. Einführung in Methodologie und Praxis qualitativer Forschung. Opladen 2000a.

Ders. (2000b): «Dokumentarische Methode». In: Hug, T. (Hrsg.): Wie kommt Wissenschaft zu Wissen? Band 3: Einführung in die Methodologie der Sozial- und Kulturwissenschaften, Baltmannsweiler.

Ders.; Loos, P.; Schäffer, B.; Städtler, K.; Wild, B. (1995): Die Suche nach Gemeinsamkeit und die Gewalt der Gruppe. Hooligans, Musikgruppen und andere Jugendcliquen im Vergleich. Opladen.

Ders. und Nohl, A. M. (1998): «Adoleszenz und Migration - Empirische Zugänge einer praxeologisch fundierten Wissenssoziologie». In: Bohnsack, R.. und Marotzki, W. (Hrsg.): Biographieforschung und Kulturanalyse. Opladen 1998.

Breitenbach, E. (2000): Mädchenfreundschaften in der Adoleszenz. Eine fallrekonstruktive Untersuchung von Gleichaltrigengruppen. Opladen.

Brown, M. E. (1994): Soap opera and women's talk. The pleasure of resistance. London u. a..

Friebertshäuser, B. und Prengel, A. (Hrsg.) (1997): Handbuch qualitativer Forschungsmethoden in der Erziehungswissenschaft. Weinheim und München.
Flick, U.; Kardorff, E.v.; Steinke, I. (Hrsg.)(2000): Qualitative Forschung. Ein Handbuch. Reinbeck

Gillespie, M. (1995): Televisions ethnicity and cultural change. London, New York.

Greenbaum, T. L. (1993): The handbook of focus group research. New York.

Günthner, S. und Knoblauch, H. (1994): «Forms are the Food of Faith. Gattungen als

Muster kommunikativen Handelns.». In: Kölner Zeitschrift für Soziologie und Sozialpsychologie Jg. 46, , H. 4, S. 693-723.

Hovland, C. I. u. a. (1949): Experiments on Mass Communication. Princeton.

Keppler, A. (1994): Tischgespräche: Über Formen kommunikativer Vergemeinschaftung am Beispiel der Konversation in Familien. Frankfurt/M.

Kromrey, H. (1986): «Gruppendiskussionen. Erfahrungen im Umgang mit einer weniger häufigen Methode empirischer Sozialwissenschaft». In: HoffmeyerZlotnik,, J. H. P. (Hrsg.): Qualitative Methoden der Datenerhebung in der Arbeitsmigrantenforschung. Mannheim.

Krueger, R. A. (1998): The focus group kit. London, New Dehli.

Lamnek, S. (1998): Gruppendiskussion. Theorie und Praxis. Weinheim.

Lazarsfeld, P. F. u. a. (1948): The Peoples Choice. New York.

Liebes. T./ Katz, E. (1990): The export of meaning. Oxford UK.

Livingstone, S. M./ Lunt, P. K. (1996): «Rethinking the Fokus Group in Media and Communications Research». In: Journal of Communication, 46 (2), , S. 79-98.

Loos, P. (1998): Mitglieder und Sympathisanten rechtsextremer Parteien. Wiesbaden.

Ders. (1999): Zwischen pragmatischer und moralischer Ordnung. Der männliche Blick auf das Geschlechterverhältnis im Milieuvergleich. Opladen.

Ders. und Schäffer, B. (2001): Das Gruppendiskussionsverfahren. Grundlagen und empirische Anwendung. Opladen

Luckmann, T. (1986): «Grundformen der gesellschaftlichen Vermittlung des Wissens: Kommunikative Gattungen». In: Neidhardt, F. u. a. (Hrsg.): Kultur und Gesellschaft. Opladen.

Maletzke, G. (1959): Fernsehen im Leben der Jugend. Hamburg.

Mangold, W. (1960): Gegenstand und Methode des Gruppendiskussionsverfahrens. Frankfurt a. M.

Ders. (1973): «Gruppendiskussionen». In: Handbuch der empirischen Sozialforschung, Bd.2. Frankfurt/M. ${ }^{3}$.

Ders. und Bohnsack, R. (1988): Kollektive Orientierungen in Gruppen Jugendlicher. Bericht für die Deutsche Forschungsgemeinschaft. Erlangen.

Mannheim, K. (1964): «Beiträge zur Theorie der Weltanschauungsinterpretation». In: Ders., Wissensoziologie. Neuwied.

Ders. (1980): Strukturen des Denkens. Frankfurt. 
Merton, R. K. und Kendall, P. L. (1946): «The focused interview». In: American Journal of Sociology 51, S. 541 - 557.

Merton, R. K. (1987): «The focused interview and focus group: Continuities and discontinuities». In: Public Opinion Quarterly, 51, S. 550 - 566.

Meuser, M. (1998): Geschlecht und Männlichkeit. Soziologische Theorie und kulturelle Deutungsmuster. Opladen.

Michel, B. (2001): «Dimensionen der Offenheit. Kollektive Sinnbildungsprozesse bei der Rezeption von Fotografien» In: Ehrenspeck, Y. und Schäffer, B. (Hrsg.): Filmund Photoanalyse in der Erziehungswissenschaft. Ein Handbuch. Opladen (i. E.)

Mikos, L. (2001): «Zur Rolle ästhetischer Strukturen in der Filmanalyse». In: Ehrenspeck, Y und Schäffer. B.: Film- und Photoanalyse in der Erziehungswissenschaft. Ein Handbuch. Opladen (i. E.)

Morley, D. (1986): Family television. Cultural power and domestic leisure. London.

Moser, H. (1999): Einführung in die Medienpädagogik. Aufwachsen im Medienzeitalter, Opladen

Nentwig-Gesemann, I. (1999): Krippenerziehung in der DDR. Alltagspraxis und Orientierungen von Erzieherinnen im Wandel. Opladen.

Nießen, M. (1977): Gruppendiskussion. Interpretative Methodologie Methodenbegründung Anwendung. München.

Nohl, A.-M. (1996): Jugend in der Migration. Türkische Banden und Cliquen in empirischer Analyse. Baltmannsweiler.

Ders. (2001): Migration und Differenzerfahrung. Junge Einheimische und Migranten im rekonstruktiven Milieuvergleich. Opladen.

Peukert, R. (1984): Gesprächs-Hermeneutik. Gruppendiskussionen als Methode zur Rekonstruktion der Lebenswelt von Lehrlingen. Band I, Frankfurt/M..

Pollock, F. (Hg.) (1955): Gruppenexperiment Ein Studienbericht, Frankfurter Beiträge zur Soziologie. Band 2, Frankfurt/M.

Schäffer, B. (1996): Die Band. Stil und ästhetische Praxis im Jugendalter. Opladen.

Ders. (1998a): «Generation, Mediennutzungskultur und (Weiter)Bildung. Zur empirischen Rekonstruktion medial vermittelter Generationenverhältnisse.»In: Bohnsack, R./ Marotzki, W. (Hrsg.): Biographieforschung und Kulturanalyse. Transdisziplinäre Zugänge qualitativer Forschung. Opladen, S. 21-50.

Ders. (1998b): «Die〈Arroganz〉 der Jüngeren? oder: Zur Bedeutung medienvermittelter Erlebniszusammenhänge für die Konstitution generationsspezifischer Erfahrungsräume.» In: Erwachsene Medien Bildung (Literatur und Forschungsreport Weiterbildung 42. Frankfurt 1998), S. 48-62.
Ders. (2000): «Das Internet: ein Medium kultureller Legitimität in Bildungskontexten?». In: Marotzki, W.; Meister, D. und Sander, U.: Zum Bildungswert des Internet. Opladen, S. 21-50.

Ders. (2001): «Generationenspezifische Wissensaneignung in einer Mediengesellschaft: das Beispiel Computerwissen». In: Beiheft zum Report (Literatur und Forschungsreport Weiterbildung) «Wissen und Lernen, didaktisches Handeln und Institutionalisierung» Dokumentation der Jahrestagung der DfG, Sektion Erwachsenenbildung in Halle vom 24. bis 26.11.2000, Opladen

Schorb, B. (1995): Medienalltag und Handeln. Medienpädagogik in Geschichte, Forschung und Praxis. Opladen

Schorb, B. (1998): «Stichwort: Medienpädagogik». In: ZfE, Zeitschrift für Erziehungswissenschaft, 1. Jg., Heft 1, S. 7-22

Schulenberg, W. (1957): Ansatz und Wirksamkeit der Erwachsenenbildung. Stuttgart.

Tully, C. J. (2000): «Jugendliche Netzkompetenz: just do it - Surfen im Cyberspace als informelle Kontextualisierung». In: Marotzki, W.; Meister, D. und Sander, U. (2000): Zum Bildungswert des Internet. Opladen, S. 189-217.

Turkle, S. (1995): Live on the Screen. Identity in the Age of the Internet. New York.

Vitouch, P. (1994): Fernsehen und Angstbewältigung, Westdeutscher Verlag.

Volmerg, U. (1977): «Kritik und Perspektiven des Gruppendiskussionsverfahrens in der Forschungspraxis». In: Leithäuser, T., u. a. (Hrsg.): Entwurf zu einer Empirie des Alltagsbewusstseins. Frankfurt/M..

Wetzstein, Th. A.; Dahm, H.; Steinmetz, L.; Lentes, A.; Schampaul, St.; Eckert, R. (1995): Datenreisende. Die Kultur der Computernetze, Opladen

Willis, Paul (1991): Jugend Stile. Zur Ästhetik der gemeinsamen Kultur. Hamburg/Berlin.

Wittpoth, J. (1994): Rahmungen und Spielräume des Selbst. Frankfurt. 